\title{
Whither the future of Pyramid City
}

\author{
Caleb Deen Bastian*1, John Stafford Bastian², and Herschel Rabitz ${ }^{1,3}$ \\ ${ }^{1}$ Program in Applied and Computational Mathematics, Princeton \\ University, Princeton, NJ. 08544, USA \\ ${ }^{2}$ Smiles For All, Hendersonville, TN. 37075 \\ ${ }^{3}$ Department of Chemistry, Princeton University, Princeton, NJ. 08544
}

April 13, 2022

\begin{abstract}
The Great Pyramids of Ancient Egypt are among the very greatest architectural achievements of humanity and are unique in their monolithic and immutable expressions. A key item is their long-term planning and maintenance as perpetuities. In view of their current state and environment and contemporary technology, resources, and ethics, we propose their complete restoration and maintenance and a collaborative decision making framework for doing so. We advance the perspective that preservation of built heritage is homologous to restoration of form and function in dentistry, and we explore how concepts in dentistry in the approach to patient casework may be applied to considerations of preservation of built heritage, vis-a-vis comprehensive treatment planning and a generalist-specialist model. Key results are existence of homology between dentistry and built heritage, and the interpretation of certain instances of built heritage as archaeo-socio-economic perpetuities. We illustrate comprehensive treatment planning for the Giza Necropolis of Ancient Egypt, containing around 10 million Pharaonic stones. Diagnosis is stone loss and chronic and acute inflammation. Comprehensive treatment planning is outlined in terms of Phases $0-3$, where 0 is emergency, 1 is information acquisition and control of pathology, 2 is restoration, and 3 is maintenance. Strictly reversible restorations are utilized in as much as Giza Necropolis is unable to give consent. The elaboration of Phase 3 for Giza Necropolis as an archaeo-socio-economic perpetuity conveys a unitary representation called 'Pyramid City.'
\end{abstract}

\section{Introduction}

We observe that conservation and preservation of existing structures are paramount issues in built heritage. They also are key issues in dentistry. In this section we advance the

* cbastian@princeton.edu 
perspective that preservation of built heritage is homologous or similar to restoration of form and function in dentistry, and we then explore how concepts in dentistry in the approach to patient casework may be applied to considerations of preservation of built heritage.

To provide a foundation to the ideas of this article with consideration of a model, we begin with mathematics. A fundamental tool or model we shall need is the stone throwing construction (STC), which constructs (or represents) objects as countable collections of points (random variables) in some space $(E, \mathscr{E})(2)$. STC forms a point process or random counting measure. A variety of operations may be performed on the underlying object of study. A key operation is marking, whereby properties or marks are identified to the points of the object in the space $(F, \mathscr{F})$. We call this marked $S T C$.

A second key tool we shall need is Oswald Spengler's proposition that cultures are superorganisms with limited and predictable lifespans over thousands of years, articulated in 1928 in his magnum opus The Decline of the West (3). Spengler identifies eight high cultures: Babylonian, Egyptian, Chinese, Indian, Mesoamerican, Classical, Arabian, and Western. A ninth would be Caral of Peru (4). Under Spengler's model, cultures are considered living organisms with lifespans averaging two millennia, first flourishing then declining.

Let us now consider built heritage stone constructions in light of these tools: built heritage stone constructions are formed by stones (points) and marked by their generative cultures. In STC notation the space $(E, \mathscr{E})$ is a stone space and the mark space $(F, \mathscr{F})$ is a culture space. Examples of stone throwing include the pyramids of Ancient Egypt and Mesoamerica, the Megalithic temples of Malta, Gobekli Tepe, etc., each having a cultural progenitor, shown below in Figure 1. Hence we think of each instance of built heritage as a collection of marked stones. A key fact about stone throwing is that stone constructions often outlast the culture itself, e.g., Pharaonic Ancient Egypt is long dead yet Giza Necropolis remains (largely) intact. These suggest the following homology.

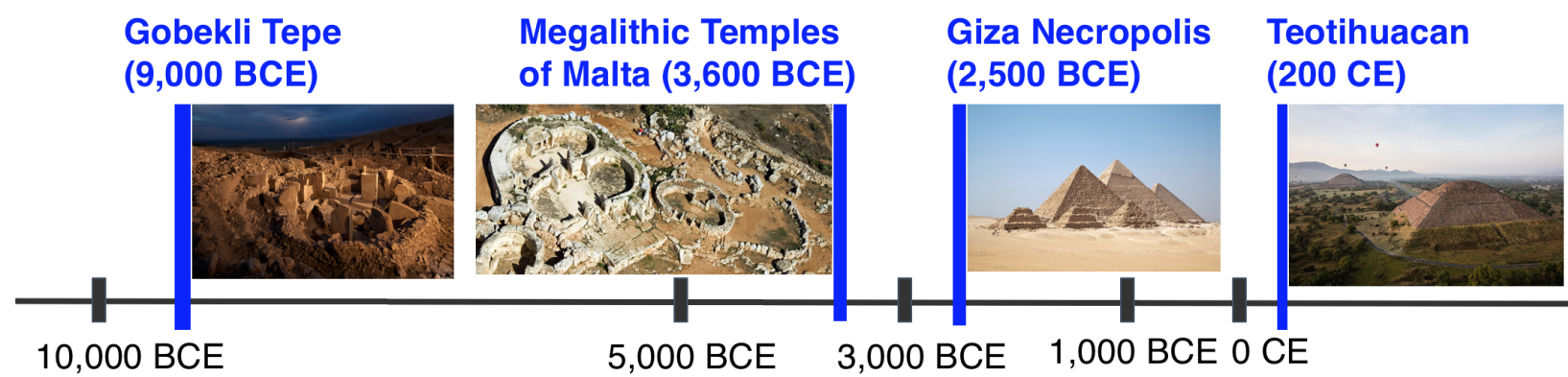

Figure 1: Timeline of 'stone throwing' cultures

A key idea is the perspective that marked STC of built heritage is similar or homologous to the marked STC of dentistry. This is seen by noting that in dentistry a dentition is formed by a collection of teeth and their adjoining structures and identified or marked by a living organism. In STC notation the space $(E, \mathscr{E})$ is a tooth space and the mark space $(F, \mathscr{F})$ is an organism space. Hence, marked STC may be used to represent dentitions of persons in terms of collections of teeth and associated structures of a living organism. A key fact about teeth is that they persist long after organism lifespan, following from the high mineral content of hydroxyapatite in enamel, cementum, and dentin. In this way, teeth are the "pennies" of the archaeological world. 
By marked STC and Spengler's model, we have established a homology between built heritage and dentistry: instances of built heritage may be regarded as teeth of cultural organisms. With homology in place, we apply key tools of dentistry - its ethical system and decision-making framework - to considerations of built heritage in order to develop comprehensive treatment plans.

The idea underlying this article is representation of stone constructions as marked point processes and discussing their homology to the marked point processes of dentitions in dental medicine. By doing so, we retrieve the ethical infrastructure of dentistry. The remainder of this article is organized as follows. In the next section (2), we describe materials and methods from dentistry. We then discuss key results from dental medicine for treatment of built heritage (3). In Section 4, we apply these ideas to a case study of the Giza Necropolis of Ancient Egypt, where we organize a four-phase comprehensive treatment plan. Phase 3 (maintenance) of the comprehensive treatment plan is elaborated by interpreting Giza Necropolis as an archaeo-socioeconomic perpetuity called 'Pyramid City.' We end with discussion and conclusions (5).

\section{Materials and methods}

Here we describe key tools of dentistry, including its ethical system, decision-making framework, and comprehensive treatment planning.

\section{$2.1 \quad$ Ethical Systems}

By establishing homology between built heritage and dentistry, the ethical infrastructure of dentistry is available to built heritage. Dentistry defines ethical principles as a universally applicable system of beliefs regarding a person's values, conduct, and ideals resulting in a definition of what constitutes proper and improper moral behavior. We briefly describe three ethical systems used in dentistry to drive decision-making: deontological, consequentialist, and virtue.

Professional obligations in dentistry are defined by deontological ethics and enumerated by a set of rules called prima facie duties, i.e. duties based on only one consideration of a single moral dimension of an action based on a single moral principle. Note that the prima facie duties are not necessarily disjoint and are distinguished from duties proper, which are duties after taking into account all relevant principles and applying some theory of how to reconcile conflict among the theories. The prima facie duties of dentistry include autonomy, beneficence, and justice. Autonomy is the duty of respect for persons and includes principles of veracity (truth-telling) and informed consent (right to make a good or bad choice). Beneficence is the duty of do good for people and includes principles of nonmaleficence (above all do no harm) and paternalism (treating a patient like a child). Beneficence goes beyond not causing harm by requiring that existing harm be removed. Justice is the duty of people in equal situations to be treated equally and includes principles of social utilitarianism (doing the most good for the most people) and fiduciary responsibility (something held in trust).

Models of professional behavior as interactions between dentist and patient are defined

by consequentialist ethics. This gives rise to the four models of guild, agent, commercial, 
and interactive. Briefly, guild is 'my way or the highway' and emphasizes the central reality of the interaction as the dentist having expertise and the patient not. Agent is 'hired gun,' where decision-making is assigned to the patient, setting aside commitment to professional values. Commercial is 'buyer beware' or 'the customer is always right' and is a competitive interaction. Interactive is the dialogue model where dentist and patient are equal partners in their relationship, each obligated to respect the other's set of values.

Normative ethics and professionalism in dentistry are defined by virtue ethics which concern state of being. These give rise to a code of professional responsibility (virtues) and typically include truthfulness, integrity, acting legally, self-responsibility, respect for others' property, treating others with dignity and respect, placing patient interest above self-interest, and promoting professional standards.

\subsection{Decision Making Framework}

The decision making framework of dentistry follows from simultaneous consideration of deontological, consequentialist, and virtue ethical systems. Therefore this framework will guide the construction of the comprehensive treatment plan for an instance of built heritage. In this article we assume an overarching interactive model between the patient and dentist. For purposes of exposition in this article, we adopt a guild model to enunciate an 'ideal' comprehensive treatment plan as a starting point for an interactive model.

\subsection{Comprehensive Treatment Planning}

Comprehensive treatment planning of dentistry generally involves organizing services and treatments into four phases, $0-3$, where 0 is emergency, 1 is information acquisition and control of pathology, 2 is restoration, and 3 is maintenance (5). The goal state is achieving "health." Under the guild model, an "ideal" comprehensive treatment plan may be developed which maximizes benefit and minimizes risk to the patient. The ideal comprehensive treatment plan restores the form and function of the dentition, i.e. ideal restoration, and assumes sufficient resources exist.

\subsection{Other Key Tools}

Other key tools are literature review, especially in research, establishing 'joins' to pertinent information, and computer-aided-design and computer-aided-manufacturing technology (CAD-CAM), bringing to bear state-of-the-art technology in information acquisition, planning, and restoration. The notion of a case study begets a health record, a structured object containing multiple types of information. These include a health history with review of systems and documentation of comorbidities and socioeconomic and psychographic factors, a model of the dentition and associated envelopes of motion, a chief complaint, diagnostic and treatment histories and values such as those conveyed through oral examinations, radiographic studies, laboratory tests, diagnostic casts, consults with specialists, CAD-CAM data, etc., one or more diagnoses as indicated by evidence, and a comprehensive treatment plan accepted by the patient under informed consent. 


\section{Results}

We summarize key results below, following from the arguments of the previous sections. The first key result is homology by marked STC.

Key Result 1 (Homology). There is homology between built heritage and dentistry in terms of marked STC, where instances of built heritage are "teeth" of cultures.

Immediately we retrieve corollaries of identifying personhood to the cultural organism, regarding each instance of built heritage as being marked with personhood.

Corollary 1 (Personhood). "Treat it as a person" - identify instances of built heritage to personhood.

Corollary 2 (Beneficence). The prima facie duty of beneficence goes beyond "do no harm" to include "remove existing harm."

Upon consideration of key constructs in dentistry, we see that the ideal comprehensive treatment plan restores and preserves the form and function of the object.

Key Result 2 (Restoration is preservation). Ideal comprehensive treatment planning implies "ideal restoration is preservation."

Key results 1 and 2 convey the central ideas of the article. A graphical depiction of these ideas is shown in Figure 2 .

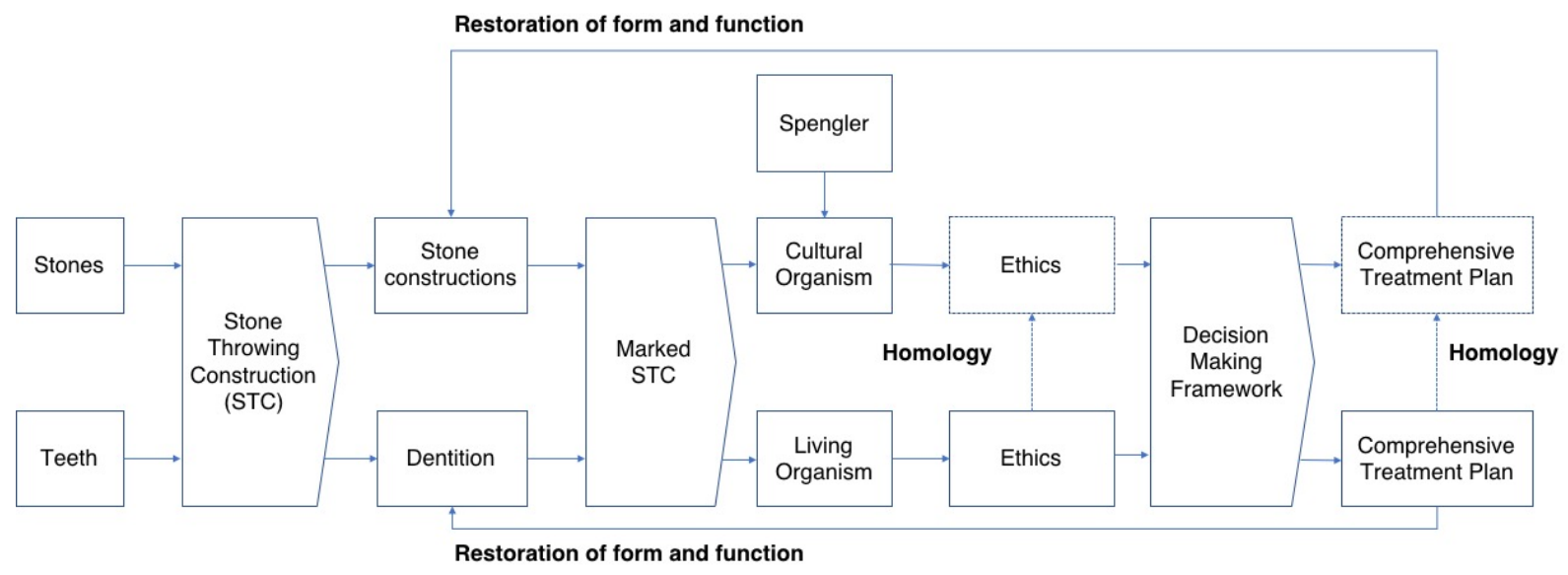

Figure 2: Graphical depiction of the main ideas of the article and their relations

The notions of form and function of instances of built heritage dates to the $19^{\text {th }}$ century ideas of French architect Eugène Viollet-le-Duc, who is regarded as the first theorist in modern architecture. Viollet-le-Duc argues that the restoration of a building "is not to maintain it, repair it or remake it: it is to re-establish it in a complete state which may never have existed at any given moment" (므). Hence Viollet-le-Duc develops a comprehensive, somewhat fanciful notion of restoration. The results of this article are generally congruent with Viollet-le-Duc's ideas. A novelty of the ideas of this article is a contemporary interpretation of form and function, where function may interpret an instance of built heritage as 
an archaeo-socio-economic perpetuity. This follows from the unique nature of personhood status conferred to the instance of built heritage through Corollary 1 and the interaction of it with socio-economic state / systems, having a perpetual lifetime. Discounted cash-flow and stochastic models may be applied to model the value of economic activities.

\section{Case Study: Giza Necropolis of Ancient Egypt}

Here we make these ideas specific by outlining a comprehensive treatment plan for Giza Necropolis of Ancient Egypt, where the objective is health, or in STC language, a nondefective measure. As a brief overview, Giza Necropolis of Ancient Egypt is a massive complex of stone funerary structures containing over 10 million stones covering an area of over 500 acres (7). It was built as a new site construction by a series of Pharaohs of the Fourth Dynasty during the Old Kingdom of Ancient Egypt over a period of 60+ years from 2,584 - 2,522 BCE, known to be Khufu, Khafre, and Menkaure. Giza Necropolis was architected as a Pharaonic solar resurrection machine and contains three main integrated pyramid complexes, one for each Pharaoh, each containing five elements (valley temple, causeway, funerary or pyramid or mortuary temple, pyramid, subsidiary pyramids). Giza Necropolis contains other features such as boat pits, mastabas (flat-topped pyramids), and enclosure walls. Note that the successful construction of Giza Necropolis was made possible by the activities of great patriarch Pharaoh Sneferu, Khufu's father, who built three pyramids himself, which may be regarded as prototypes to those of Giza Necropolis. In addition, Giza Necropolis contains the Great Sphinx, Sphinx temple, and an additional pyramid complex of Queen Khentkaus (Khentkawes) I. In the original setting, the three pyramid complexes of Khufu, Khafre, and Menkaure had functional harbors with boats docking near the valley temples. Giza Necropolis is renowned as the greatest, oldest, and only remaining Wonder of the Ancient World.

Figures 3 and 4 show depictions of Giza Necropolis in present day. 


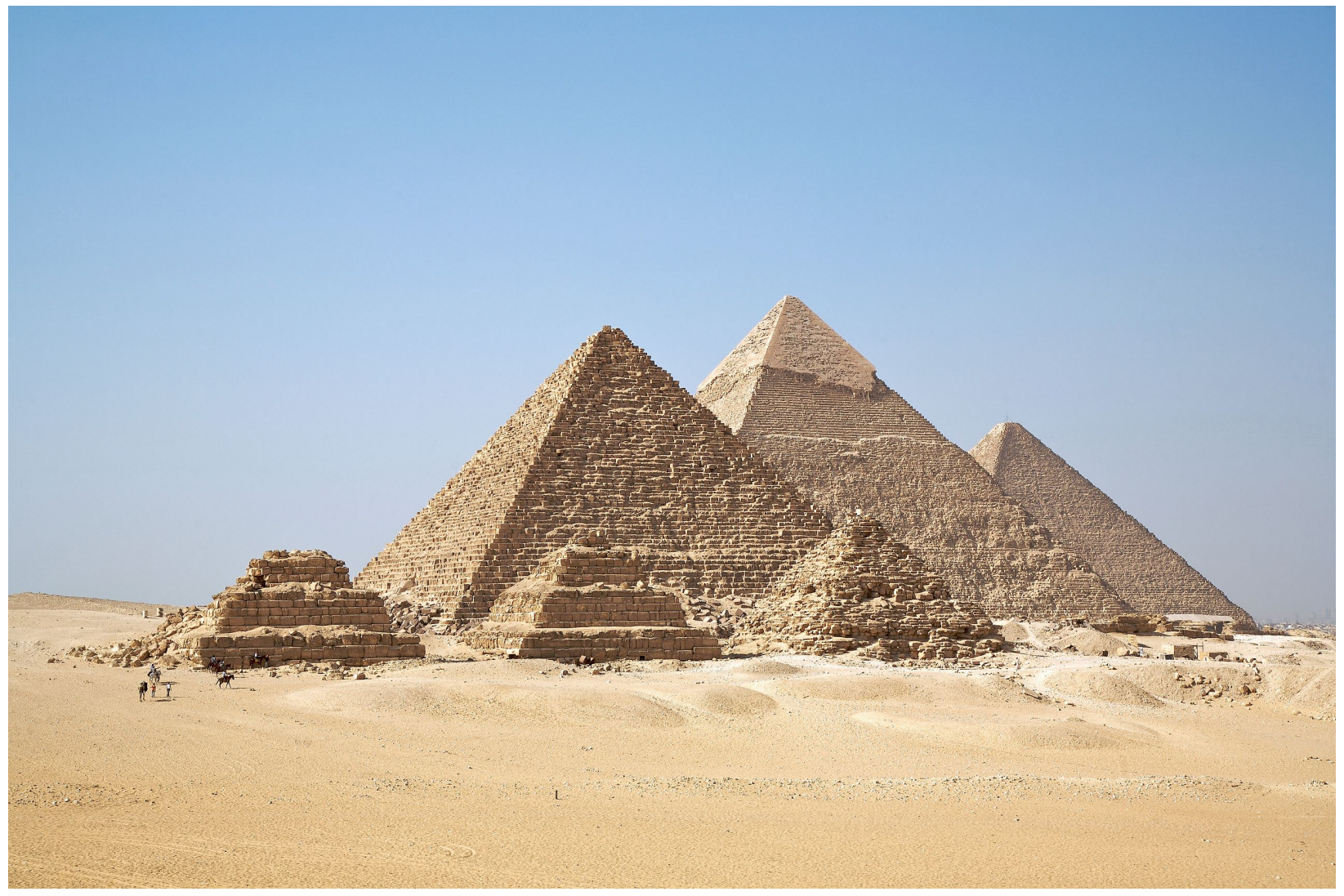

Figure 3: A representative image of Giza Necropolis in present day, the 'pyramids shot' (8) 


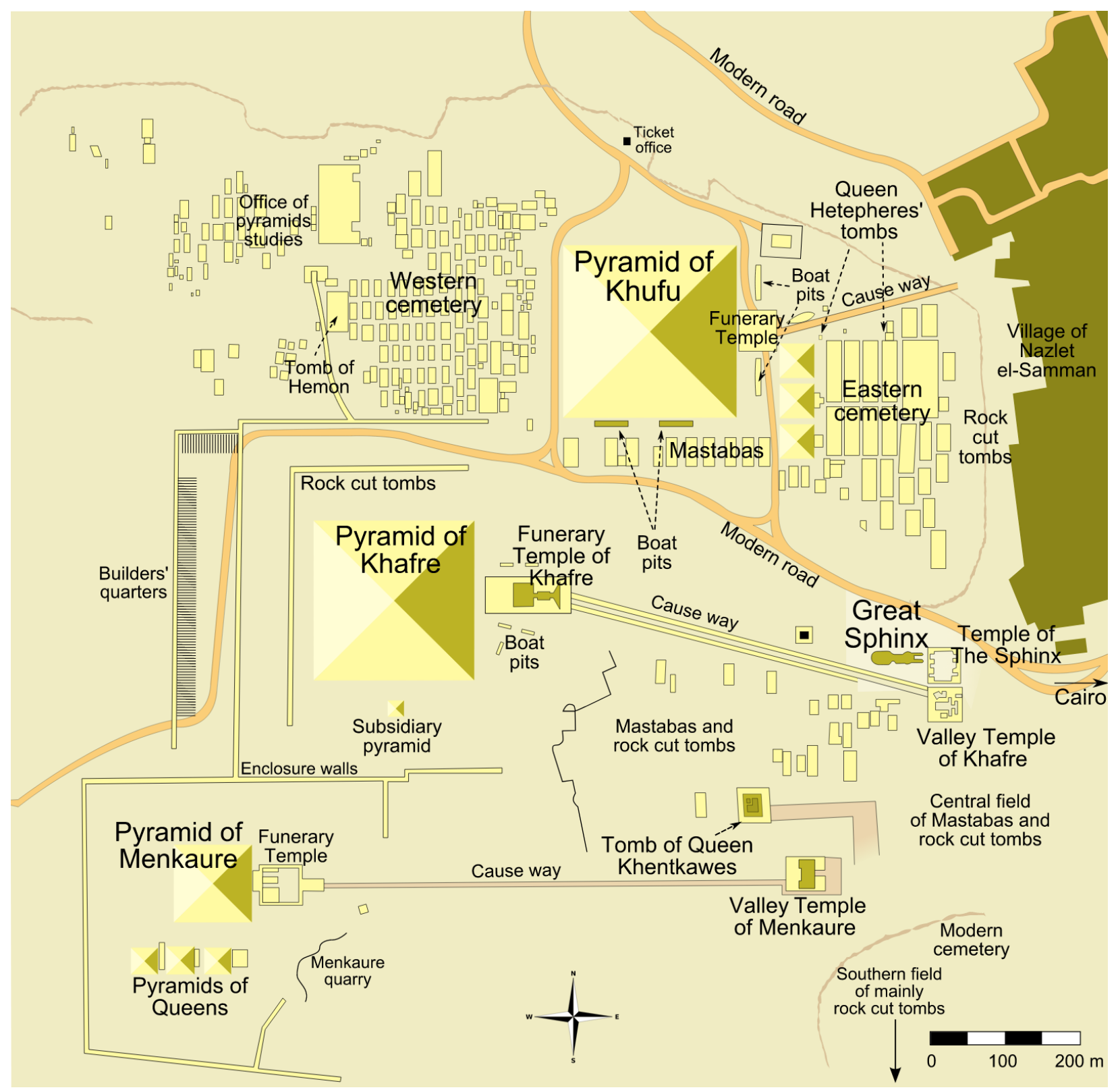

Figure 4: Annotated map of Giza Necropolis in present day (Wikipedia 2019) (8) 


\subsection{Model}

First, to give a proper model of Giza Necropolis, we use STC, i.e. Giza Necropolis exists as a collection of 10 million stones and their Pharaonic marks. Consider measurable space $(E, \mathscr{E}) \subset\left(\mathbb{R}^{3}, \mathscr{B}_{\mathbb{R}^{3}}\right)$ and Pharaonic mark space $(F, \mathscr{F})$. Pharaoh Khufu and his family managed for realization $\omega \in \Omega$ (Pharaonic STC)

$$
M_{\omega}(A)=\sum_{i=1}^{K(\omega)} \mathbb{I}_{A}\left(X_{i}(\omega), Y_{i}(\omega)\right)=\sum_{i=1}^{10^{7}} \mathbb{I}_{A}\left(X_{i}(\omega), Y_{i}(\omega)\right) \quad \text { for } \quad A \in \mathscr{E} \otimes \mathscr{F}
$$

where $M$ formed by $(\mathbf{X}, \mathbf{Y})$ is a magnification of that formed by the stones $\mathbf{X}$. In modern day stones are missing, where some of the $\mathbf{X}$ are located at a 'cemetery' $\partial$, some point outside of $E$. Therefore $M_{\omega}$ is defective on $(E \times F, \mathscr{E} \otimes \mathscr{F})$ with defect $D(\omega)=K(\omega)-M_{\omega}(E \times F)$, corresponding to the lost stones, on order of one percent of the total stone count.

\subsection{Materials}

Interior stones are limestone quarried locally on-site. The casing stones are either polished Turah limestone or Aswan granite, depending on the pyramid. The pavement about the pyramids is basalt. Statuary is generally made of diorite or graywacke.

\subsection{Charting}

Figure 3 contains key features and landmarks. The book Giza and the Pyramids by Hawass and Lehner gives detailed description of the site (7).

\subsection{Health history}

We cite historiographies of Giza Necropolis (7) and Ancient Egypt (9) and of Old Kingdom administration and economy (10; 11).

\subsection{Informed Consent}

The patient Giza Necropolis is unable to give consent (Ancient Egypt is long dead). Therefore the prima facie duty of beneficence following from nonmaleficence implies that all restoration should be completely reversible. The custodian of Giza Necropolis is the Arab Republic of Egypt.

\subsection{Generalist - specialist model}

The case is driven by a generalist who works with a number of specialists. Specialists include Egyptologists, material science engineers, land and waterworks engineers, economists, technologists (CAD/CAM), supply chain professionals, and so on and so forth. There will be a number of consults made with specialists relative to each phase. 


\subsection{Diagnosis}

Stone loss and chronic and acute inflammation

\subsection{Aesthetics}

The three aesthetic principles of Giza Necropolis (and more generally the Fourth Dynasty) are clarity, power, and sophistication. Immense spans of huge stones, extending many hundreds of feet, assume triangular form in facets, with a necklace of structures reposed on the ground, each collection distinct, while unified in order. The three smooth-sided pyramids of Giza Necropolis convey near perfect isotropy of triangular form in the surfaces with immense dimensions. For reference, the slant height of Khufu's pyramid is 612 feet, with base 756 feet and height (altitude) 481 feet, and the base hypotenuse is 1,069 feet. The funerary and valley temples are masterpieces of megalithic stone throwing, akin to a dance of 'Legos' of stone. Giza Necropolis also possesses a degree of self-similarity, with arrays of small pyramids nested within an array of large pyramids. Khufu's mastabas are large and organized, laid out in a grid-like fashion. The pyramids are smooth-sided with surfaces comprised of polished Turah limestone (white) or Aswan granite (pink). The effect is that they reflect light. The pyramids had pyramidions (tips of the pyramids), sometimes covered in electrum or having inscriptions in hieroglyphics. Statuary is known for Khafre in diorite "Khafre enthroned" and for Menkaure in the graywacke triads, enabling some degree of identification and reproduction. Long causeways connect the pyramid and valley temples, similar to the causeway of the Pyramid of Unas, remnants of which exist today. Integration of harbors confers vitality of an aquatic setting, with flora and fauna. The aesthetics of Giza Necropolis follow from the composition of these various elements.

\subsection{Idealized comprehensive treatment plan}

(if sufficient resources exist) Using STC, we describe the superposition of old stones and new stones, where independent new Pharaonic stones are made in the number $D(\omega)$ as (Pharaonic $\mathrm{STC})$

$$
M_{\omega}^{*}(A)=\sum_{i=1}^{D(\omega)} \mathbb{I}_{A}\left(X_{i}(\omega), Y_{i}(\omega)\right)=\sum_{i=1}^{10^{5}} \mathbb{I}_{A}\left(X_{i}(\omega), Y_{i}(\omega)\right) \quad \text { for } \quad A \in \mathscr{E} \otimes \mathscr{F}
$$

Then

$$
N_{\omega}=M_{\omega}+M_{\omega}^{*}
$$

is the superposition of the old stones $M_{\omega}$ and the new stones $M_{\omega}^{*}$ with $N_{\omega}(E \times F)=K(\omega)$, i.e., $N_{\omega}$ is a non-defective magnification on $(E \times F, \mathscr{E} \otimes \mathscr{F})$.

Note that the figures do not depict modern improvements that are nearby but are unobtrusive, including the Grand Museum of Egypt and the museum for Khufu's solar barge.

\section{Comprehensive Treatment Plan, Phases $0-3$}

Phase 0 (emergency) Control of 'acute inflammation' - remove trash and debris from Giza Necropolis. 
Phase 1 (information acquisition and control of pathology) Build models for Giza Necropolis and its adjoining land as they are today and through restoration. Excavate the entire Necropolis and remove all sand from the area (storage off-site). Clean all surfaces as appropriate. Control of 'chronic inflammation' of encroaching development: use imminent domain to condemn and remove a defined adjoining region to Giza Necropolis, with relocation of places, and land engineering towards the original and natural setting of a harbor. Control of pathological processes such as erosion and reconstruction of original flood control systems.

Numerous consults will be required in Phase 1. Land and waterworks engineers will provide insight into restoring the site and adjoining area to its original condition. Presently there is much development that has encroached upon Giza Necropolis, so economists will provide insight into purchasing the land condemned by imminent domain. Egyptologists will be consulted, and all excavations will be under their purview.

Phase 2 (restoration) Complete and perfect restoration of Giza Necropolis. New stone will be provisioned such that the original surface morphology is conveyed. All stone will be reversibly stacked to ensure complete reversibility of restoration. Land and waterworks engineering will recreate the natural harbor setting.

Numerous consults will be found in Phase 2 restoration efforts, including material science engineers for measurable material properties and assessments of their impact when interfacing with the current in situ stone. Egyptologists will be consulted on stone and surface finish decisions, including the pyramidions, statuary, and reliefs.

Phase 3 (maintenance) Regular cleanings, comprehensive inspections, etc., into perpetuity. This could entail creation of a suitable independent institution for these responsibilities.

Estimated Cost (by phase) Phase 0 costs are small in comparison to later phases. Say $\$ 100$ million USD for thorough cleaning to relieve acute inflammation.

Phase 1 costs will be dominated by the cost of purchasing condemned land. Clearly the magnitude of land purchase will depend upon its scope. Land and waterworks engineering will also be a significant undertaking. Excavation is comparatively less expensive. Say $\$ 1$ billion USD for relieving chronic inflammation.

Phase 2 costs will be dominated by the raw materials and transportation costs of the new stones and conveyance to their positions in the edifice. Say $\$ 1$ billion USD for replacing the lost stone.

Phase 3 costs will be dominated by creating and endowing institutions. Say $\$ 1$ billion USD endowment to pay for maintenance and cleaning into perpetuity.

This gives a total cost of $\sim \$ 3$ billion USD for a comprehensive treatment plan for Giza Necropolis.

Risk analysis (by phase) Phase 0 is very low risk, e.g., removing acidic bat feces from pyramid interiors.

Phase 1 is low risk, where risk comes from the actions of removing and demolishing modern development, including roads, parking lots, buildings, etc., where present day de- 
velopment has consumed Khufu's causeway and valley temple and Khufu's, Khafre's, and Menkaure's harbors. Modern technology confers low risk to these activities.

Phase 2 is low risk, where risk comes from routine handling of stone, e.g., stone slippage, dropping, etc. Reversible stone stacking means that every restoration of surface morphology can be perfectly undone.

Phase 3 is low risk provided that suitable controls on handling of funds are implemented.

Benefit analysis (by phase) Phase 0 benefits are clean surfaces for the stone, no odor, etc.

Phase 1 benefits are reclaiming key components of Giza Necropolis and protecting the site from development.

Phase 2 benefit is complete and reversible restoration of the surfaces.

Phase 3 benefit is Giza Necropolis being maintained in splendid form.

Below in Figure 5 we give an entity-relationship diagram (graph) for the comprehensive treatment plan for Giza Necropolis where arrows indicate information flow.

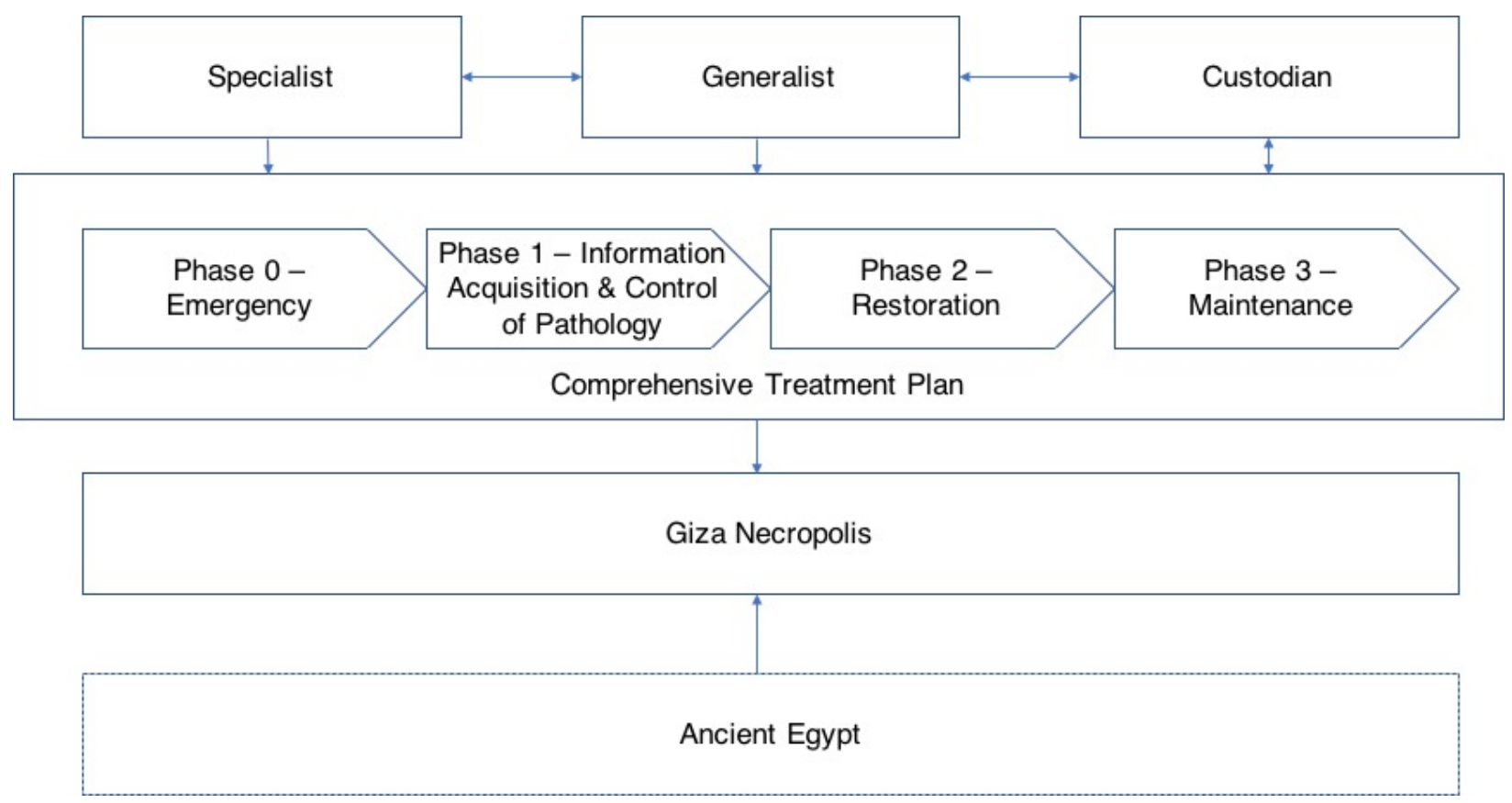

Figure 5: Summary of comprehensive treatment plan for Giza Necropolis of Ancient Egypt

In the following figures, we give a visual and graphic overview of the ideas contained in this article regarding preservation as restoration and maintenance of form and function relative to the Giza Necropolis of Ancient Egypt. We show existing and proposed form and function in paired water-color paintings. 


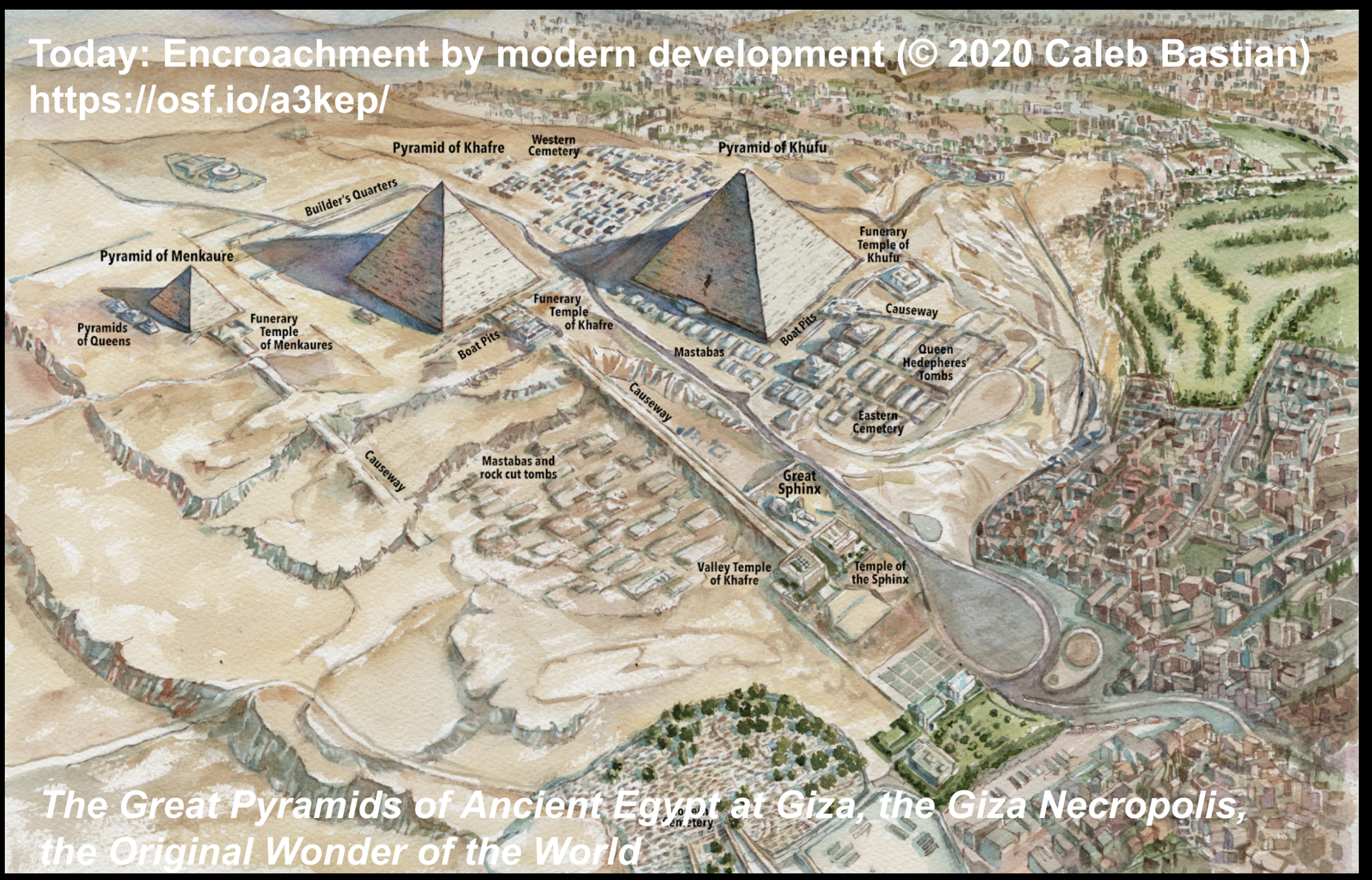

The Giza Necropolis, today 


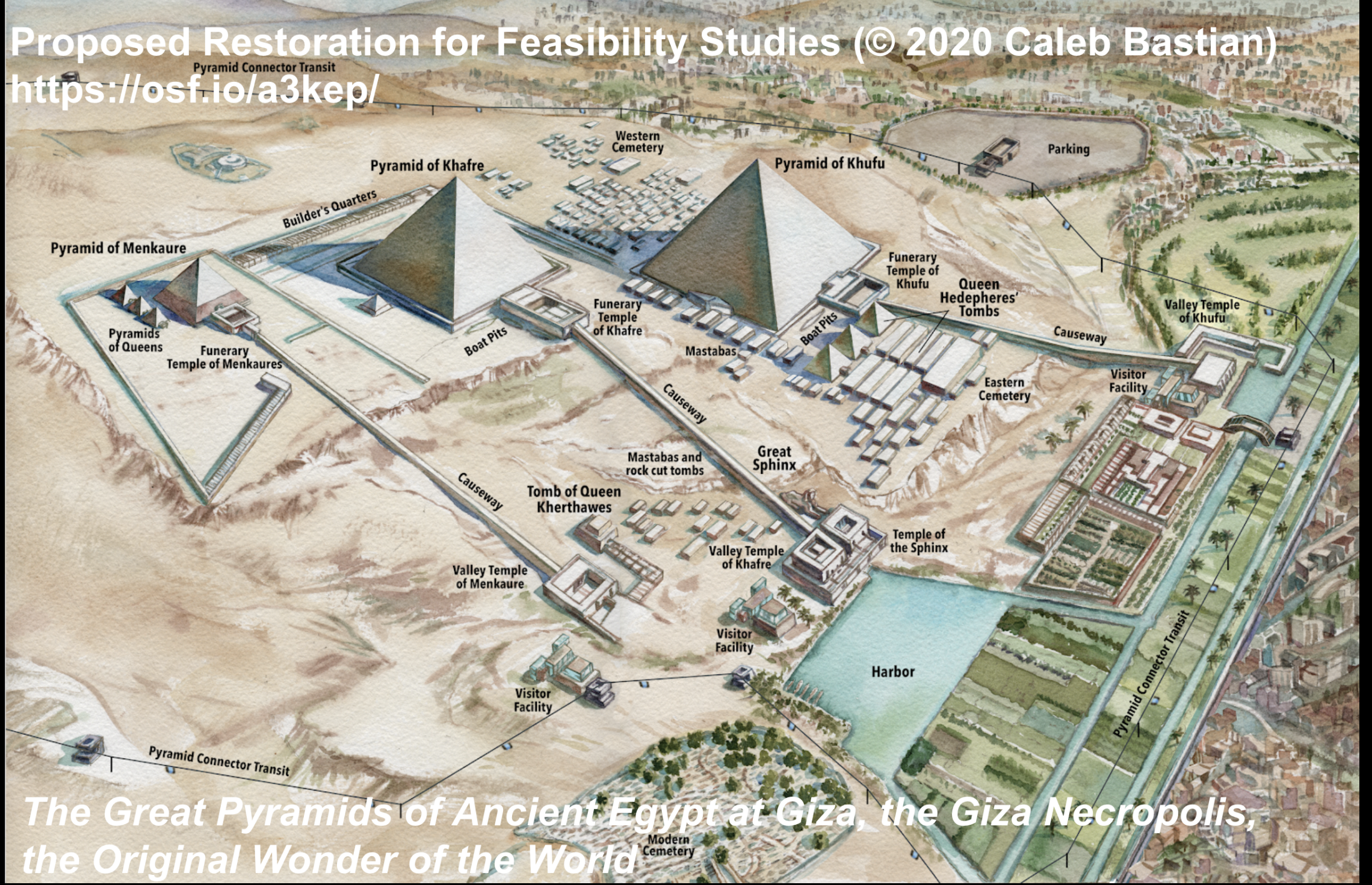

The Giza Necropolis under comprehensive treatment planning 


\subsection{Elaboration of Phase 3 Treatment Planning: 'Pyramid City'}

Giza Necropolis is a perpetual institution. Moreover, it interfaces with the state of Egypt, including its society, and the world, e.g., tourism. Looking far to the future, comprehensive treatment planning (Phase 3) of Giza Necropolis as an archaeo-socio-economic perpetuity ('Pyramid City' or 'The Solar City') would provide a structured and natural means to mediate these relations indefinitely. Here, Giza Necropolis, and more generally the Pyramid Fields, is accorded an expanded form of legal status, with, in an imaginative telling, domain and accoutrements of a city-state, which we call, in view of its context, Pyramid City (PC). The basis of the action of creating PC is to put in place the necessary conditions for carrying out comprehensive treatment planning of the Giza Necropolis and the larger collection of the Pyramid Fields into perpetuity. There are multiple motivations for and benefits of such a proposition, which we discuss below.

First, Giza Necropolis is the maximum statistic of Ancient Egypt and the cultural dominator and progenitor of all that came after. It is the most well known, oldest, largest, best preserved, and most celebrated of the major Ancient Egyptian sites. It is the archetype par excellence of the Egyptian pyramid complex, a form subsequently reproduced dozens of times. Through its comprehensive treatment planning, the necessary resources and strategies to entertain a larger theater of such efforts for the Pyramids Fields are thus primed.

Second, the task of comprehensive restoration and maintenance of the Pyramid Fields is colossal: there are roughly 30 pyramids and other structures located over a 70 mile corridor from Abu Rawash to the Fayoum. This concentration of riches is in a favorable configuration: all the sites are co-located, a 'stones throw' away from one another, such that the task tremendously benefits from economies scale and scope. Execution of comprehensive treatment planning over such a large and unique domain necessitates large resources and specialized administration. Such resources and administration must be centralized, independent, and absolute, to achieve the necessary efficiency, effectiveness, and credibility to sustain the effort. All such considerations are folded into the definition of PC.

Third, the design for and control of safe ingress and egress to PC, functioning as an eternally open 'park,' must be a careful undertaking. As a guiding principle, there should never a threat to the integrity of the structures caused by human presence. From this principle, basic constraints follow. A simple zero-order constraint is a capacity factor on the total number of visitors allowed into the park at any point in time. First-order constraints are individual capacity factors applied to park subdivisions. And so on and so forth. The park is a collection of nodes (sites) that form a straight-line path over mostly barren sand on a plateau. A simple, unobtrusive, multi-nodal transportation network is a system of high-speed gondolas, here appointed with air-conditioning, wireless internet, etc. A gondola moving at 30 miles per hour could traverse the entire park in one direction in a little over 2 hours. Each connection between nodes takes around 5 minutes. Each node of the park, identified to one or more sites (clusters), could have individually designed and carefully located improvements, such as a visitor facility, museum, restaurants, shops, etc, perhaps partially subterranean to minimize height. The improvement types and their distribution over the park would need careful study. Audio-visual shows and other entertainment could be incorporated.

Fourth, in possession of domain, such as some of the undeveloped desert to the west of the 
Pyramid Fields, PC could create zoning for residential, commercial, educational, financial, and administrative development. These would include for example living space and markets for residents and visitors, hotels / resorts, a university, research institutes, an airport, and healthcare and financial systems.

Fifth, PC would bring a manifold of benefits to Egypt. Egypt would see its crown jewels burnished, polished, and endowed. Egypt would directly financially benefit by its Treasury receiving ongoing transfer payments from $\mathrm{PC}$ as part of a revenue share. Egypt would also see substantial improvements to its gross domestic product and to its balance of payments, following (foreign) direct investment, economic development, and foreign currency in-flows. PC would operate markets for locally made (Egyptian) goods and services, redirecting flows of resources into Egypt. It would strengthen security through economic prosperity. The creation of PC enriches Egypt's representation and in the context of global leisure and tourism economies would lead to greater liquidity and economic wealth.

Sixth, the experience of PC could benefit other sites, too, in pursuing comprehensive treatment plans, e.g., reconstruction of the Pharos Lighthouse of Alexandria, a large-scale stone depiction and Wonder of the Ancient World. The steady improvement of the fortunes of PC could culminate in establishing through comprehensive treatment planning another archaeo-socioeconomic perpetuity adapted relative to the Theban Necropolis ('Thebes City'). Here the Memphis and Theban Necropolises represent Lower and Upper Egypt respectively. These entities could interact and have various reciprocities for activities.

We graphically depict the nodal layout of Pyramid City in Figures 8 and 9 . 


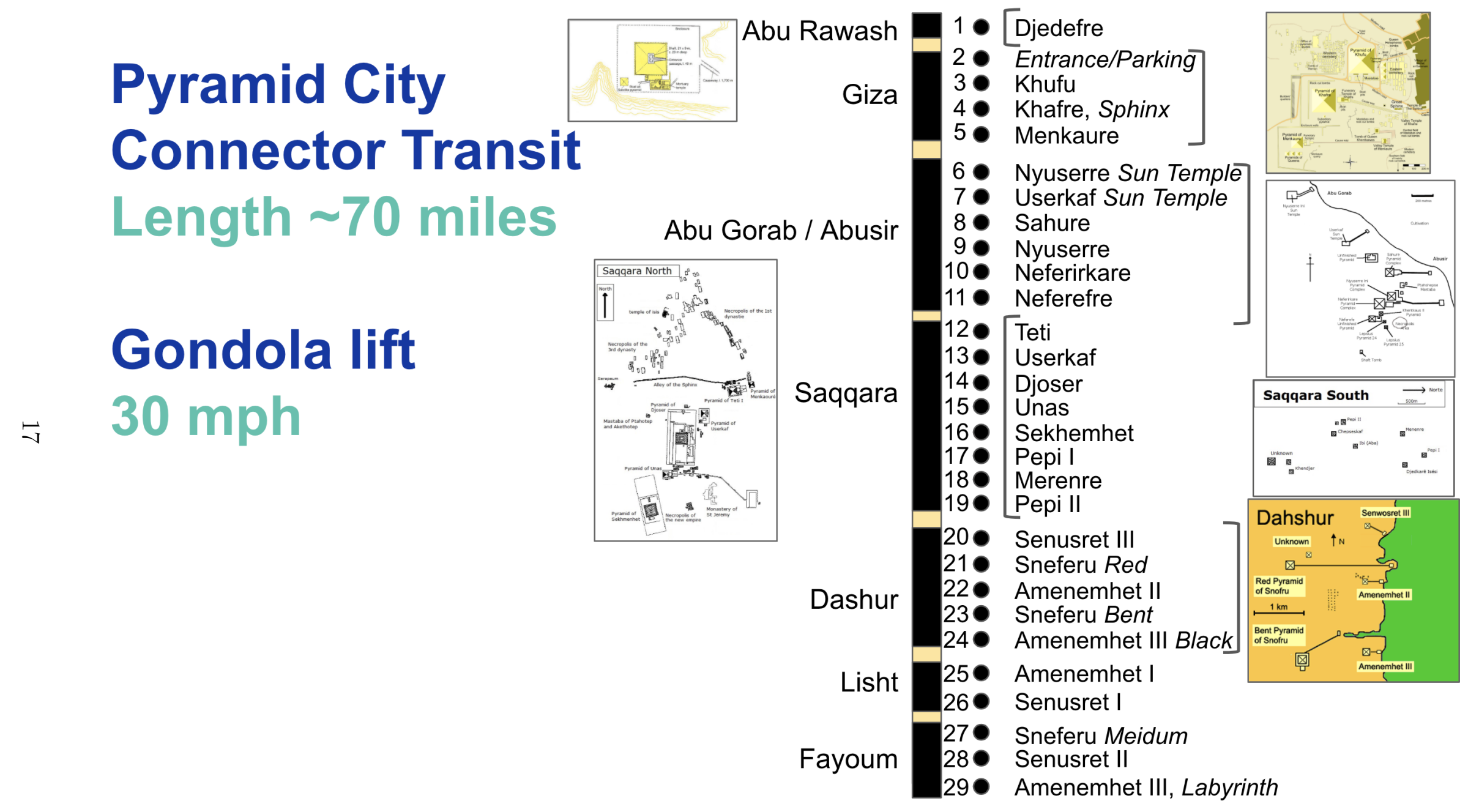

Figure 8: Pyramid City 'Park' Connector Transit (Historic sites) 


\section{Pyramid City Connector Transit}

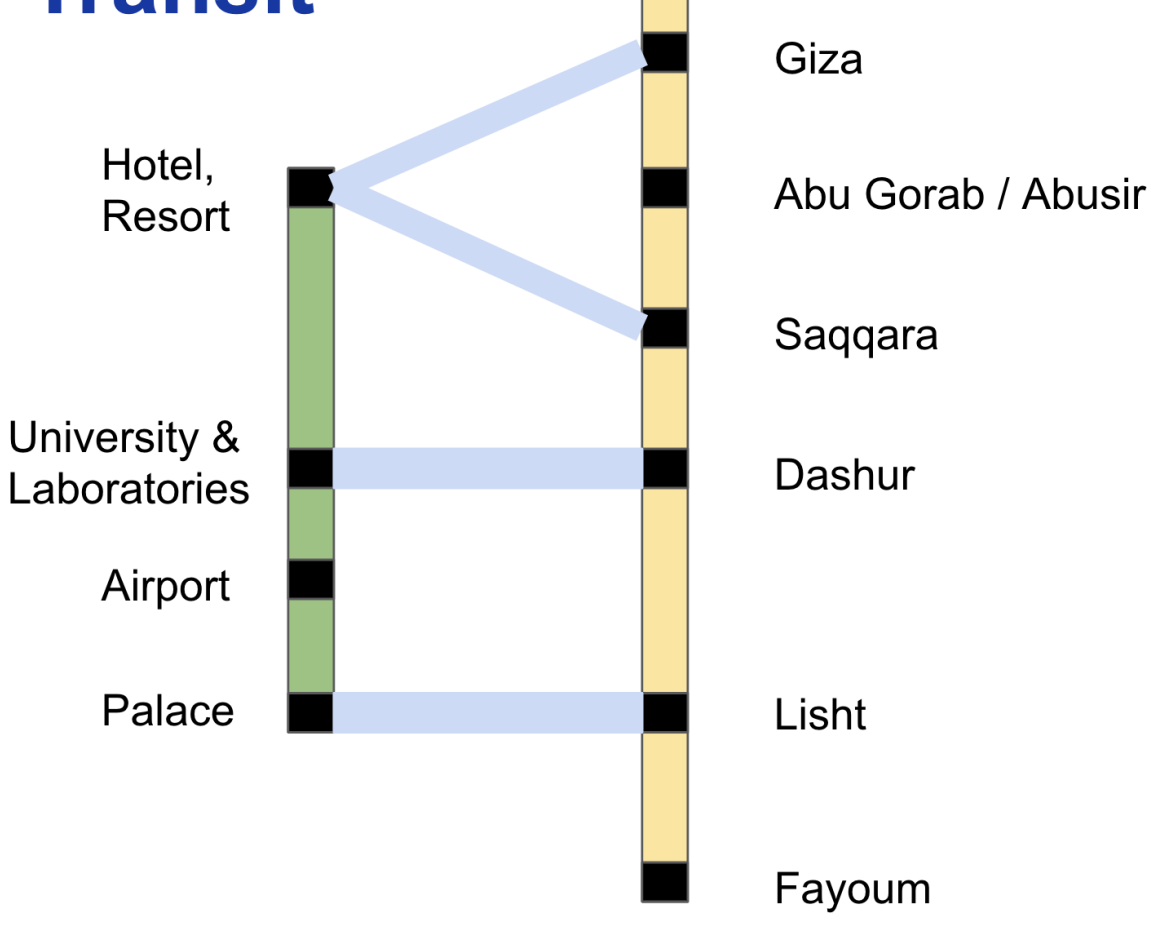

Figure 9: Pyramid City Connector Transit (Development and Park) 


\section{Discussion and Conclusions}

First, we have discussed a general concept from measure theory in mathematics called (marked) stone throwing construction (STC), which we use as a characterizing model for both dentistry and built heritage.

Second, we have accepted the hypothesis of Spengler's model of cultures as organisms, which establishes personhood of cultures. By marked STC, this induces a homology between dentistry and built heritage.

Third, we employ key tools of dentistry to outline a comprehensive treatment plan for built heritage, including phase $0-3$ planning, consults, generalist-specialist interactions, etc. As a case study we outlined a comprehensive treatment plan for Giza Necropolis (GN) of Ancient Egypt. We utilized strictly reversible restorations, following from observation that GN is unable to give consent. We have described costs, benefits, and risks of the comprehensive treatment plan by phase and procedure, making it actionable (fundable) and feasible.

Fourth, through fashioning of a comprehensive treatment plan (here outlined), we encode the complete information (necessary and sufficient) to achieve a controlled transition from present state ruins to fully restored complexes, maintaining form indefinitely.

Fifth, we may utilize STC to study objects having 'marks' or properties. For the case study, we suppose each stone of GN has a 'Pharaonic' cultural mark. Then the process of the stones is automatically adapted to the flow of information generated by the stones and is independent of all other processes. In a way, restoring GN using STC rekindles the flame of Ancient Egypt.

Sixth, these ideas lay the foundations for further elaborations of the comprehensive treatment plan. For the case study, phase 3 (maintenance) may identify GN as an archaeo-socioeconomic perpetuity called Pyramid City. This confers novel meaning to the institutions mentioned in phase 3.

\section{Declaration of Interest}

The authors report no interests.

\section{References}

[1] Goeree JK, Holt CA. Coordination Games. In: Moortgat M, editor. Encyclopedia of Cognitive Science. Macmillan Reference Ltd; 2001. .

[2] Bastian C, Rempala G. Throwing Stones and Collecting Bones: Looking for Poisson-like Random Measures; 2018. arXiv:1807.09684 [math.PR].

[3] Spengler O. The Decline of the West. New and revised ed. ed. New York : A. A. Knopf; 1928. Available from: https://search.library.wisc.edu/catalog/ 999866633402121. 
[4] Haas J, Creamer W, Ruiz A. Dating the Late Archaic occupation of the Norte Chico region in Peru. Nature. 2004;p. 1020-1023.

[5] Stefanac S, Nesbit S. Diagnosis and Treatment Planning in Dentistry. St. Louis: Mosby; 2017.

[6] Viollet-le-Duc E. Dictionary of French architecture from 11th to 16th century. vol. 8; 1854.

[7] Lehner M, Hawass Z. Giza and the Pyramids. University of Chicago Press; 2017.

[8] Wikipedia. Giza pyramid complex; 2019 [cited February 23, 2019]. Available from: https://en.wikipedia.org/wiki/Giza_pyramid_complex.

[9] Kemp B. Ancient Egypt: Anatomy of a Civilization. London: Routledge; 2006.

[10] Strudwick N. The Administration of Egypt in the Old Kingdom. KPI Limited; 1985.

[11] García JCM, editor. Ancient Egyptian Administration. vol. 104 of Handbook of Oriental Studies. Section 1 The Near and Middle East. Brill; 2013.

[12] Golvin JC. Jean-Claude Golvin; 2019 [cited February 24, 2019]. Available from: http: //jeanclaudegolvin.com.

[13] Hawass Z. Site management at Giza Plateau: master plan for the conservation of the site. International Journal of Cultural Property. 2000;9(1):1-22. Available from: https://www.cambridge.org/core/article/ site-management-at-giza-plateau-master-plan-for-the-conservation-of-the-site/ OB117ECD6D43CD3363C4E80541DF447F.

[14] Warne K. A Voice for Nature. National Geographic. 2017;

[15] Steele JM. Stochastic Calculus and Financial Applications. Springer-Verlag, New York; 2001.

[16] Bednarek Z, Patel P, Ramezani C. Sharpe Ratio over investment Horizon. California Polytechnic State University; 2004.

[17] Anderson JE. The Gravity Model. National Bureau of Economic Research Working Paper Series. 2010 December 2010;No. 16576. Available from: http://www.nber.org/ papers/w16576.

[18] Data Bank. World Bank; 2019.

[19] Balance of Payments Manual. International Monetary Fund; 2018.

[20] Rabitz H, Alis OF. General foundations of high dimensional model representations. Journal of Mathematical Chemistry. 1999;25(2-3):197-233. Available from: http:// dx.doi.org/10.1023/A\%3A1019188517934. 